\title{
Lactose-derived oligosaccharides in the milk of elephants: comparison with human milk
}

\author{
Clemens Kunz ${ }^{1}$, Silvia Rudloff ${ }^{1}$, Wolfgang Schad ${ }^{2}$ and Daniel Braun ${ }^{2}$ \\ ${ }^{1}$ Research Institute of Child Nutrition, Heinstück 11, 44225 Dortmund, Germany \\ ${ }^{2}$ Institute for Evolutionary Biology and Morphology, University of Witten/Herdecke, Stockumer Str. 10, \\ 58448 Witten, Germany
}

(Received 24 July 1998 - Revised 29 March 1999 - Accepted 11 May 1999)

\begin{abstract}
Human milk is commonly considered to be unique when compared with the milk of other species with regard to its high content of complex fucosylated and sialylated lactose-derived oligosaccharides. We describe the application of high-pH anion-exchange chromatography with pulsed amperometric detection and TLC to characterize and quantitate neutral and sialylated lactose-derived oligosaccharides in milk from three Asian elephants and human milk. The lactose contents of elephant and human milks were $25-30 \mathrm{~g} / \mathrm{l}$ and about $66 \mathrm{~g} / \mathrm{l}$ respectively, whereas total oligosaccharide concentration was about three times higher in elephant milk and comprised up to $40 \%$ ( $10 \%$ in human milk) of the carbohydrate content. The ratio neutral : acidic components was different in the milk of the two species; in elephant milk, the $N$-acetylneuraminic acidcontaining oligosaccharides made up almost half of the total amount $v .30 \%$ in human milk. Most oligosaccharides in elephant milk were more fucosylated and/or sialylated compared with human milk components. By mild acid hydrolysis, fucose and $\mathrm{N}$-acetylneuraminic acid were cleaved off from complex components, and this resulted in increased amounts of fucose, galactose, $N$-acetylneuraminic acid, lactose and lacto- $N$-neo-tetraose. Unique to elephant milk are the high levels of $3^{\prime}$-galactosyllactose (up to $4 \mathrm{~g} / \mathrm{l}$ ) and lacto- $N$-neo-tetraose which are present in human milk only in trace amounts. Elephant and human milks have high levels and unique patterns of oligosaccharides which may reflect the relative importance of these components in neonatal host defence, in endothelial leucocyte interactions or in brain development.
\end{abstract}

Elephant milk: Breast milk: Oligosaccharides

Milk from most terrestrial species contains lactose as the main carbohydrate. Exceptions are milks from monotremes (echidna, platypus (Ornithorhyncus anatinus)) and marsupials such as the tammar wallaby (Macropus eugenii) and others which contain little lactose, but a few small oligosaccharides (Gross \& Bolliger, 1958; Bergmann \& Housley, 1968; Messer \& Mossop, 1977). According to Messer \& Kerry (1973) the main components of echidna milk are sialyllactose $(50 \%)$ and fucosyllactose $(25 \%)$, and that of platypus milk is difucosyllactose. These small oligosaccharides are also common in human milk throughout lactation (Egge, 1993; Montreuil, 1993). The main carbohydrate in milk of the tammar wallaby has been identified as a trisaccharide (3'-galactosyllactose), at up to $16 \mathrm{~g} / 1$ (Messer et al. 1980). This component is present in human milk only in trace amounts. However, human milk is commonly considered to be unique when compared with that of eutherian species with regard to its high content of complex fucosylated and sialylated oligosaccharides (Egge, 1993; Kunz \& Rudloff, 1993). Meanwhile, more than 130 different components of human milk have been characterized (Kobata, 1977; Egge, 1993; Montreuil, 1993; Kunz et al. 1996a). An elongation of core structures with up to twenty lactosamine units has been reported (Stahl et al. 1994). Some of these components are discussed as being involved in the infant's defence system, the development of a specific intestinal microflora and in inflammatory processes (Kunz et al. 1993; Zopf \& Roth, 1996; Kunz, 1999). To date, no other milk has been reported to resemble human milk both in quantity and quality of the oligosaccharide fraction, including the milk of rhesus monkeys (Macaca mulatta) (Kunz et al. 1996b). With regard to elephant milk

\footnotetext{
Abbreviations: HM, human milk sample; HPAEC-PAD, high-pH anion-exchange chromatography with pulsed amperometric detection: HPTLC, highperformance thin-layer chromatography; LNFP, lacto- $N$-fucopentaose; LNT, lacto- $N$-tetraose.

* Corresponding author: Dr Clemens Kunz, fax +499939 049, email clemens.kunz@eruaehrung.uni-giessen.de
} 
composition, only a few studies have been performed, and these have demonstrated that, for example, the fatty acid pattern is highly unusual compared with milks from other species (McCullagh et al. 1969).

Here, we describe the application of high-pH anionexchange chromatography with pulsed amperometric detection (HPAEC-PAD) and high-performance TLC (HPTLC) to characterize and quantitate neutral and sialylated lactose-derived oligosaccharides in milk from three Asian elephants (Elephas maximus) which turned out to be highly unusual regarding both composition and concentration of oligosaccharides when compared with any other milk examined so far.

\section{Materials and methods}

\section{Materials}

Milk samples (1-5 ml) from three Asian elephants (45-234 d post partum) were donated from the Zoo Carl Hagenbeck (Hamberg-Stellingen, Germany). The samples were taken, without prior oxytocin injection. by hand-milking immediately after the young had stopped sucking. Mature human milk samples $(10-50 \mathrm{ml})$ from 2 to $28 \mathrm{~d}$ post partum were obtained from healthy, exclusively breast-feeding mothers from the Children's Hospital, Dortmund, Germany. Human milk was completely expressed manually or by a breast pump and a portion was taken and frozen until analysis.

Oligosaccharides which had been isolated from human milk and characterized in earlier studies (Egge et al. 1983; Kunz et al. 1996a) were used as standards. Other standards were obtained from BioCarb (Lund, Sweden) or Oxford Glycosystems (Cambridge, UK).

Bio-liquid chromatography was performed on a DionexSystem (Sunnyvale, CA, USA) consisting of a CarboPac PA-1 column $(250 \times 4.6 \mathrm{~mm}$ i.d. $)$ equipped with a guard column and a Model PAD 2 detector. $\mathrm{NaOH}$ solution ( $19 \mathrm{~mol} / \mathrm{l}$; low in carbonate) was purchased from Baker (Philadelphia, PA, USA). Sodium acetate of analytical grade was from Merck (Darmstadt, Germany). Sephadex G25 was obtained from Pharmacia (Uppsala, Sweden). Thin-layer plates (Silica-gel $60,100 \times 100 \mathrm{~mm}$ ) were purchased from Merck. All other reagents were of analytical grade.

\section{Comparative analysis}

To get a first impression of the differences in the carbohydrate composition of milk from human subjects and elephants $1 \mathrm{ml}$ whole milk was first defatted by centrifugation at $3000 \mathrm{~g}$ for $20 \mathrm{~min}$ at $4^{\circ}$ (Kunz et al. 1996a). After the lipid layer had been removed and the aqueous phase decanted and filtered through glass wool, $660 \mathrm{ml} / 1$ precooled ethanol $(950 \mathrm{ml} / \mathrm{l})$ was added to precipitate proteins. Then, ethanol was extracted by flushing with $\mathrm{N}_{2}$ before direct analysis of the carbohydrate fraction by HPTLC and HPAEC-PAD (see later).

\section{Isolation of oligosaccharides}

The first steps for the preparation of milk for oligosaccharide analysis were accomplished as described previously by
Kunz et al. (1996a). Fat was removed as described earlier and the aqueous phase decanted and filtered through glass wool. Proteins were precipitated by adding an equal volume of precooled acetone, stirring for $2 \mathrm{~h}$ and keeping overnight at $4^{\circ}$. Most of the lactose in the supernatant fraction was removed by repeated crystallization in a rotary evaporator (Egge et al. 1983). The residual fraction was applied to a Sephadex G25 column $(900 \times 25$ mmi.d. $)$ and eluted with water. Carbohydrate-containing fractions were identified by the anthrone (neutral components) and Ehrlich methods (acidic components (Svennerholm, 1957; Dische, 1962). Fractions with residual peptides (ninhydrin-positive fractions) were excluded from the analysis. Then, salt-free but carbohydrate-positive fractions were lyophilized before further analysis. These fractions sometimes contained small amounts of lactose which is difficult to separate completely from oligosaccharides due to the large quantity in milk and its similar molecular mass compared with other small milk oligosaccharides.

\section{High-performance TLC}

Defatted and deproteinized milk samples $(5 \mu \mathrm{l})$ were diluted with $15 \mu \mathrm{l}$ water; then, $2 \mu \mathrm{l}$ was applied to silica-gel HPTLC. Lyophilized fractions (1 mg) after Sephadex G25 gel filtration chromatography were dissolved in $1000 \mu \mathrm{l}$ water; then $15 \mu l$ was analysed by silica-HPTLC in butanol-acetic acid-water $(2 \cdot 5: 1: 1$, by vol.) (two subsequent runs) or butanol-ethanol-water-acetic acid-pyridine ( $5: 50$ : $15: 1 \cdot 5: 5$, by vol.). The plates were sprayed with orcinol for carbohydrate detection $\left(1 \mathrm{ml}\right.$ orcinol/l in $\left.2.3 \mathrm{M}-\mathrm{H}_{2} \mathrm{SO}_{4}\right)$ or ninhydrin $(0.3 \mathrm{~g}$ ninhydrin in $95 \mathrm{ml}$ 2-propanol containing $5 \mathrm{ml} 960 \mathrm{ml} / \mathrm{l}$ acetic acid) for peptides.

\section{High-pH anion-exchange chromatography with pulsed amperometric detection}

The system and the separation conditions have been described previously in detail (Kunz et al. 1996a). Briefly, neutral and acidic oligosaccharides were analysed using the following conditions: eluent A, $100 \mathrm{mM}-\mathrm{NaOH}$; eluent B, $100 \mathrm{~mm}-\mathrm{NaOH}$ and $250 \mathrm{~mm}$-sodium acetate. The elution programme began with $3 \mathrm{ml}$ eluent $\mathrm{A}$, followed by a gradient of up to $100 \%$ eluent $\mathrm{B}$ in $30 \mathrm{~min}$. A re-equilibration volume of $5 \mathrm{ml}$ eluent A was chosen. The flow rate of $1.0 \mathrm{ml} /$ min was used and $25 \mu \mathrm{l}$ from $1 \mathrm{mg} / 250 \mu \mathrm{l}$ or $1 \mathrm{mg} / 1000 \mu \mathrm{l}$ solutions was injected.

\section{Release of fucose and $\mathrm{N}$-acetylneuraminic acid}

Cleavage of $N$-acetylneuraminic acid and fucose by mild acid hydrolysis was carried out as described previously (Hardy et al. 1988). Lyophilized Sephadex G25 fractions were resuspended in water $(1 \mathrm{mg} / 100 \mu \mathrm{l})$ and $10 \mu \mathrm{l}$ transferred into Wheaton tubes. $\mathrm{H}_{2} \mathrm{SO}_{4}(0 \cdot 05 \mathrm{M}, 50 \mu \mathrm{l})$ was added to each tube under $\mathrm{N}_{2}$. The samples were mixed and incubated for $1 \mathrm{~h}$ at $80^{\circ}$. Then, $0 \cdot 1 \mathrm{M}-\mathrm{NaOH}$ was carefully added to the cooled tubes until neutral conditions were achieved $(25-30 \mu \mathrm{l})$. Cleaved $N$-acetylneuraminic acid and fucose were detected in the hydrolysed sample by HPAEC-PAD (Kunz et al. 1996a). 


\section{Quantitation of lactose and oligosaccharides}

Lactose was determined photometrically after enzymic conversion (kit from Boehringer Mannheim, Germany). Oligosaccharides were quantified after HPAEC-PAD analysis. Standard oligosaccharides (Oxford Glycosystems, Oxford, UK) were used to determine molar response factors in relation to the abundant milk oligosaccharide lacto- $N$-tetraose (LNT). Peak areas of all oligosaccharides to be quantified were then calculated as a percentage of the total amount of oligosaccharides applied, corrected by the corresponding factor for the molar response of the amperometric detector.

\section{Results}

HPTLC of defatted and deproteinized milk showed that the total carbohydrate content was much higher in elephant milk compared with human milk (Fig. 1). This observation was confirmed by HPAEC-PAD of the same samples. Fig. 2 demonstrates that many components which are listed in the legend were common to both species although the number of neutral and acidic components as well as the oligosaccharide pattern differed markedly.

To identify and characterize lactose-derived oligosaccharides in milk from elephants the fat- and protein-free milk was subjected to Sephadex G25 gel filtration followed by HPAEC-PAD of each fraction. The large variety of lactose-derived oligosaccharides in elephant milk is demonstrated in Fig. 3(a-d). Neutral oligosaccharides elute from the column at between 1 and 17 min whereas the retention time of acidic components is $>17 \mathrm{~min}$. Most oligosaccharides are highly fucosylated and/or sialylated, and this can easily be demonstrated by releasing fucose and $N$-acetylneuraminic acid by mild acid hydrolysis of the Sephadex G25 fractions. The overlays in Fig. 3 show the mono- and oligosaccharide compositions before (bottom chromatogram) and after (upper chromatogram) hydrolysis. $N$-acetylneuraminic acid is cleaved off from acidic components such as $3^{\prime}$ sialyllactose, $6^{\prime}$-sialyllactose and sialyllacto- $N$-tetraose a and c resulting in large lactose and free $N$-acetylneuraminic acid

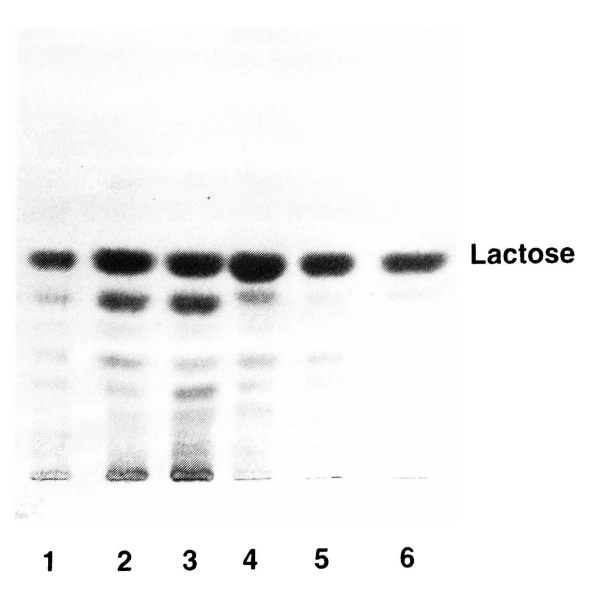

Fig. 1. Silica-high-performance TLC of defatted and deproteinized milk samples from three elephants and three women. Portions $(5 \mu \mathrm{l})$ of defatted and deproteinized milk were diluted with $15 \mu \mathrm{l}$ water; then, $2 \mu \mathrm{l}$ was applied to the plate. For details of procedures, see pp. 392-393. Lanes 1-3, elephant milk; lanes 4-6, human milk. peaks (Fig. 3(a and b)). The fucose peak in Fig. 3(a and b) reveals that many sialylated components are also fucosylated. Fractions (c) and (d) contained mainly neutral components as demonstrated by the appearance of fucose, galactose and lactose after acid hydrolysis.

In milk from elephants there was one major component with a retention time of $15 \cdot 2$ min using HPAEC-PAD (Figs. 2 and $3(d)$ ). This peak, which was mainly found in the lactose fraction after Sephadex G25 gel filtration (Fig. 4), was manually collected and subjected to silica-HPTLC to check the purity (Fig. 5). NMR analysis revealed that this component was $\alpha 3$-galactosyllactose which in human milk is only found in trace amounts. Its concentration can be as high as $13 \mathrm{~g} / \mathrm{l}$ in elephant milk.

Quantitative analysis revealed that the lactose content in elephant milk was less than half that of human milk whereas the total oligosaccharide concentration was about three times higher in elephant milk (Table 1) and made up to $40 \%$ ( $10 \%$ in human milk) of the carbohydrate content. The ratio neutral : acidic components was significantly different

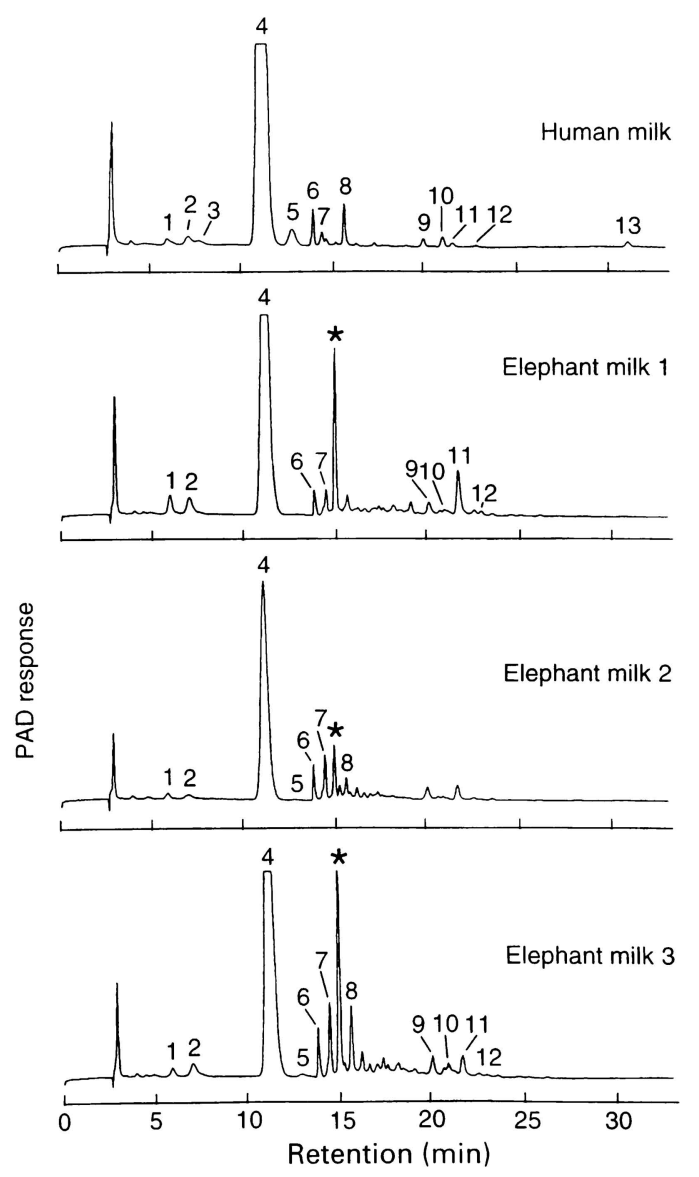

Fig. 2. High-pH anion-exchange chromatography with pulsed amperometric detection (PAD) of defatted and deproteinized milk samples from three elephants and one woman. Human milk was obtained on day 28 , elephant milks, 1,2 and 3 on days 234,45 and 72 post-partum respectively. Peak designation: (1) lacto- $N$-difucohexaose I; (2) 3-fucosyllactose; (3) lacto- $N$-fucopentaose II; (4) lactose; (5) 2'-fucosyllactose; (6) lacto- $N$-fucopentaose I; (7) lacto- $N$-neotetraose; (8) lacto- $N$-tetraose; (9) sialyllacto- $N$-tetraose c; (10) $6^{\prime}$ sialyllactose; (11) $3^{\prime}$-sialyllactose; (12) sialyllacto- $N$-tetraose a; (13) disialyllacto- $N$-tetraose; $\left(^{*}\right) 3^{\prime}$ galactosyllactose. 


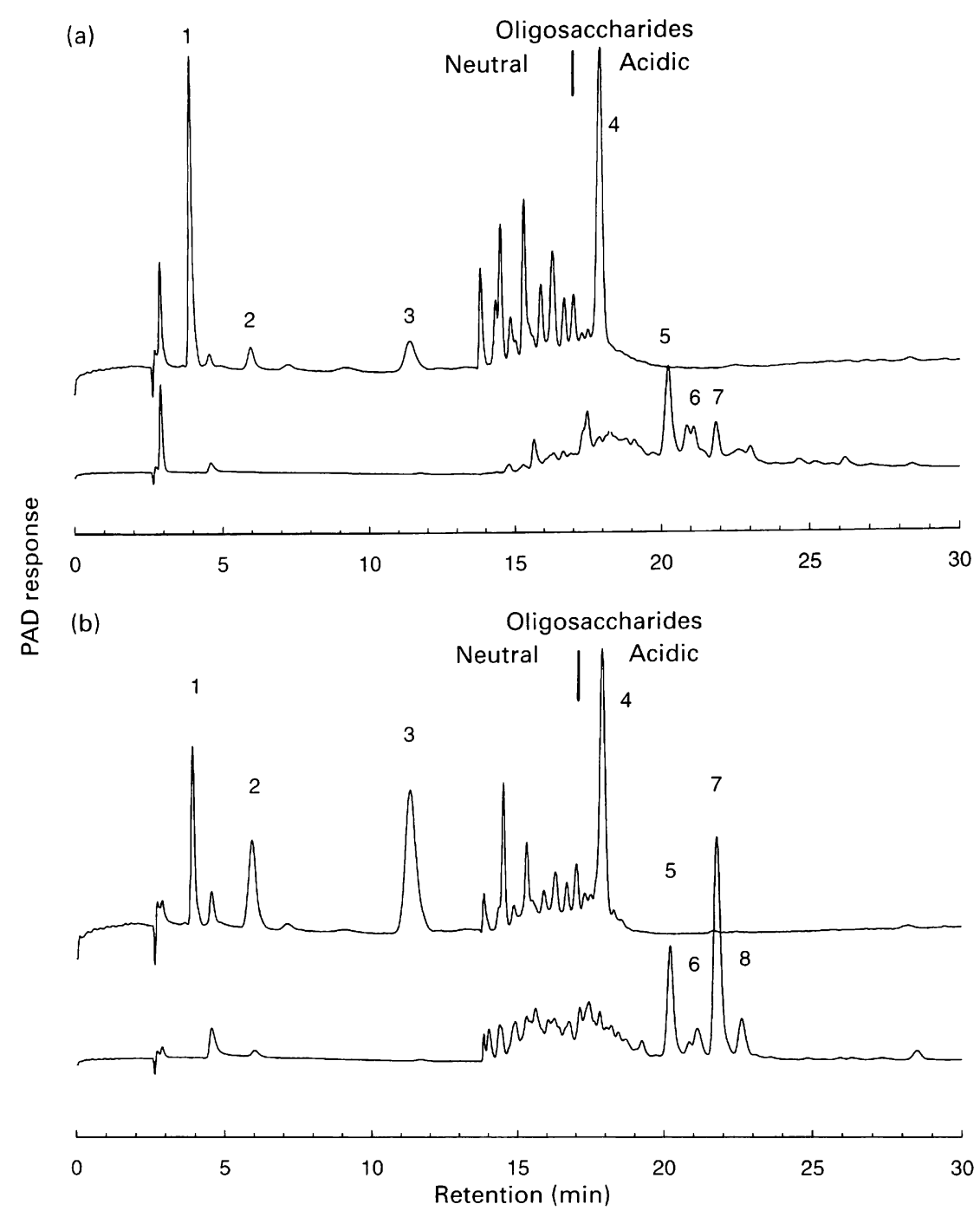

Fig. 3. High-pH anion-exchange chromatography with pulsed amperometric detection (HPAEC-PAD) of carbohydrate-positive fractions a-d after Sephadex G25 gel filtration of defatted and deproteinized elephant milk. To remove fucose and/or $\mathrm{N}$-acetylneuraminic acid, the fractions were subjected to mild acid hydrolysis and subsequently analysed by HPAEC-PAD. In the overlays, the samples before (bottom chromatogram) and after (upper chromatogram) mild acid treatment are shown. Peak designation: (1) fucose; (2) galactose; (3) lactose; (4) $\mathrm{N}$-acetylneuraminic acid; (5) sialyllacto- $\mathrm{N}$-tetraose c; (6) 6'-sialyllactose; (7) 3'-sialyllactose; (8) sialyllacto- $N$-tetraose a; (9) lacto- $N$-neotetraose; (10) $\alpha$-galactosyllactose.

in the milk of the two species: in human milk, only one-third of the total amount was acidic oligosaccharides; in elephant milk, however, the sialic-acid-containing oligosaccharides made up almost half of the total amount. In addition, these acidic components contained many more fucose residues than in human milk.

Quantification of individual components such as $2^{\prime}$ fucosyllactose, LNT and lacto- $N$-fucopentaose 1 (LNFP) revealed that these were major neutral oligosaccharides present in human milk which were also present in elephant milk in varying concentrations (Table 1). It is also evident that the concentration of lacto- $N$-neo-tetraose was very high in elephant milk whereas only traces could be detected in human milk. Among sialylated oligosaccharides, the concentrations of $3^{\prime}$-sialyllactose and sialyllacto- $N$-tetraose c were much higher in elephant milk than in human milk.
With regard to changes in oligosaccharide pattern and content we analysed eighteen milk samples from day 2 to day 19 post partum from four women (Table 2). Due to their structural similarity many oligosaccharides showed similar retention times on HPAEC-PAD. Therefore, we chose only those components which could be quantified without any difficulties.

Among the neutral oligosaccharides LNT, LNFP I and LNFP II were the major components (Table 2). Although LNT increased in milk of all mothers during the first few days post partum, the intra- and interindividual variation was high with a range of $0 \cdot 12 \mathrm{~g} / \mathrm{l}$ (human milk sample (HM) 3) to $1.6 \mathrm{~g} / \mathrm{l}(\mathrm{HM} \mathrm{1)}$. It is striking that the concentrations of LNT in HM 1 and 2 were about twice those in HM 3 and 4.

With regard to the pattern of two monofucosylated LNT derivatives, the concentration of LNFP I exceeded that of 
(c)

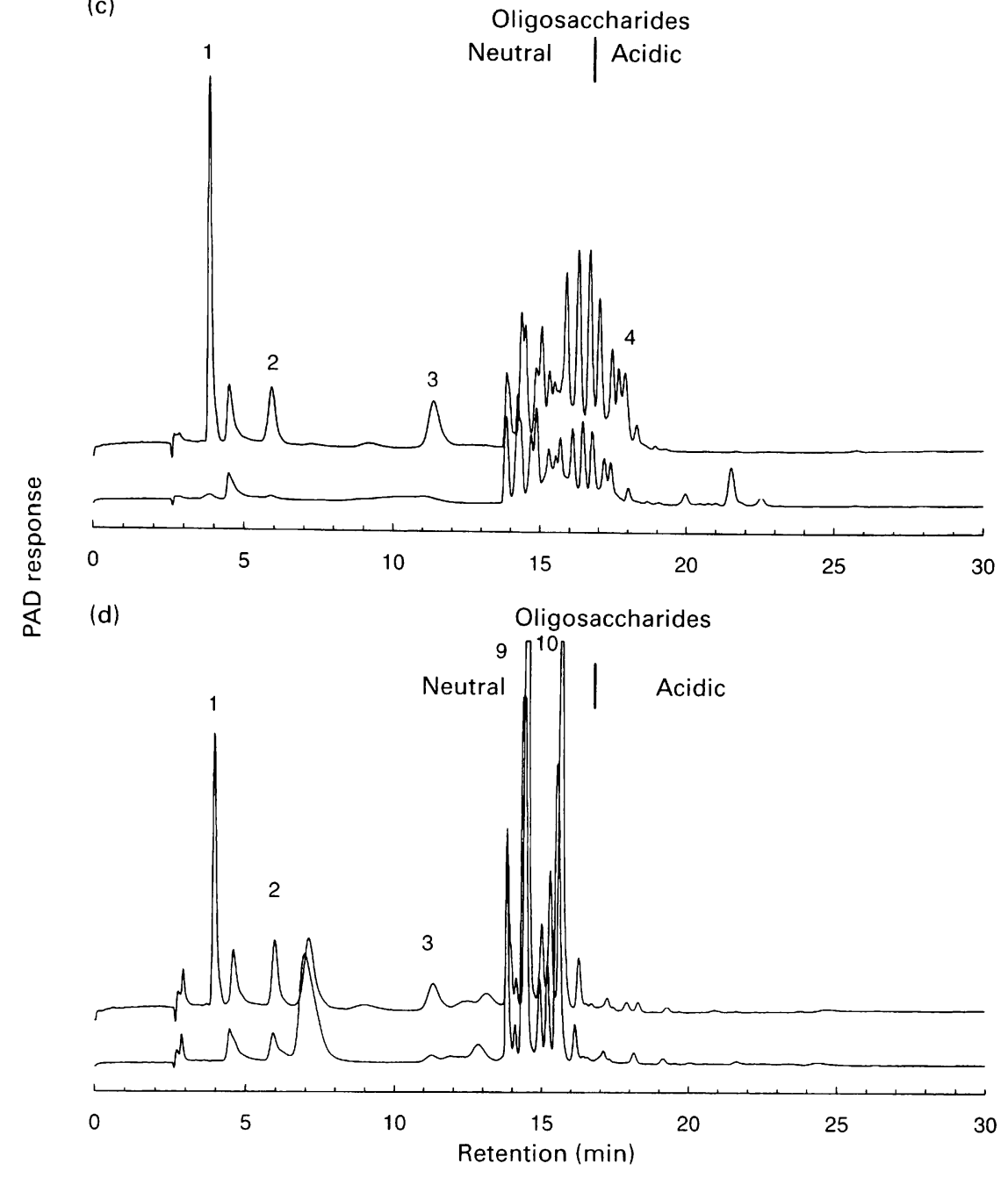

Fig. 3 (continued).

Table 1. Concentrations of lactose and oligosaccharides $(\mathrm{g} / \mathrm{l})$ in elephant milk $(\mathrm{EM})^{\star}$ and human milk (HM) $\dagger$ (Mean values, with standard deviations for $\mathrm{HM}$ )

\begin{tabular}{|c|c|c|c|c|c|}
\hline \multirow[b]{2}{*}{ Component } & \multirow[b]{2}{*}{ EM1 } & \multirow[b]{2}{*}{ EM2 } & \multirow[b]{2}{*}{ EM3 } & \multicolumn{2}{|c|}{$\mathrm{HM}(n 10)$} \\
\hline & & & & Mean & SD \\
\hline Lactose & $25 \cdot 77$ & $30 \cdot 67$ & $29 \cdot 60$ & $66 \cdot 47$ & 3.91 \\
\hline Neutral oligosaccharides & $10 \cdot 40$ & $11 \cdot 15$ & $13 \cdot 15$ & $5 \cdot 75$ & 0.59 \\
\hline 3'-Galactosyllactose & 2.97 & $1 \cdot 21$ & $4 \cdot 00$ & ND & ND \\
\hline $2^{\prime}$-Fucosyllactose & ND & ND & ND & 0.45 & 0.43 \\
\hline 3-Fucosyllactose & 0.26 & 0.16 & 0.31 & 0.07 & 0.08 \\
\hline Lacto-N-tetraose & 0.50 & 0.87 & 0.24 & 1.09 & 0.47 \\
\hline Lacto-neo- $N$-tetraose & $1 \cdot 80$ & $1 \cdot 62$ & 0.79 & $\mathrm{Tr}$ & $\mathrm{Tr}$ \\
\hline Lacto- $N$-fucopentaose I & 0.94 & $1 \cdot 26$ & 0.37 & $1 \cdot 26$ & $1 \cdot 11$ \\
\hline Difucosyllacto- $N$-hexaose II & $0 \cdot 11$ & 0.22 & 0.05 & $0 \cdot 16$ & $0 \cdot 11$ \\
\hline Sialylated oligosaccharides & $10 \cdot 60$ & $8 \cdot 10$ & $8 \cdot 63$ & $2 \cdot 10$ & 0.37 \\
\hline $3^{\prime}$-Sialyllactose & $1 \cdot 89$ & $0 \cdot 86$ & $2 \cdot 79$ & 0.27 & 0.08 \\
\hline 6'-Sialyllactose & $0 \cdot 13$ & 0.34 & 0.24 & 0.38 & 0.05 \\
\hline Sialyllacto- $N$-tetraose a & 0.52 & $0 \cdot 15$ & 0.05 & 0.14 & 0.05 \\
\hline Sialyllacto- $N$-tetraose $\mathrm{C}$ & 1.09 & 0.39 & 0.36 & 0.17 & 0.11 \\
\hline Total oligosaccharides & $21 \cdot 00$ & $19 \cdot 25$ & $21 \cdot 78$ & $7 \cdot 85$ & 0.61 \\
\hline
\end{tabular}

ND, not detectable; $\mathrm{Tr}$, trace.

*Milk from three elephants from days 45 (EM 1), 234 (EM 2) and 72 (EM 3) post partum respectively.

† Human milk from 2 to 4 weeks post partum. 


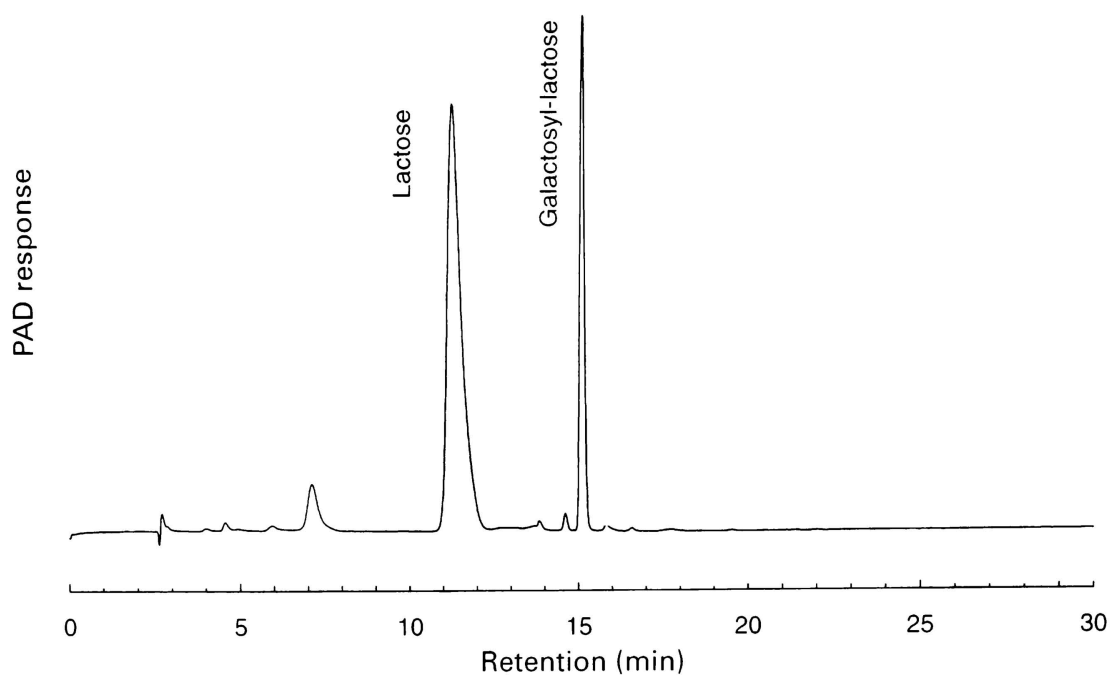

Fig. 4. High-pH anion-exchange chromatography with pulsed amperometric detection of fraction E from elephant milk after Sephadex G25 gel filtration, containing lactose and an unknown component which was later identified as $\alpha$-galactosyllactose.

LNT in HM 3 and 4 with values of up to $2 \mathrm{~g} / \mathrm{l}$. Its concentration was already very high in milk at the beginning of lactation. In HM 1 and 2, however, this component was only present in trace amounts. LNFP II, another monofucosylated LNT derivative, was only detectable in HM 1 and 2. Here, its concentration increased during lactation. In contrast to LNFP I, however, the concentration of LNFP II did not reach the level of LNT.

The changes during lactation in the acidic components were not as pronounced as in the neutral fraction. The concentration of neuraminyl- $\alpha 2-6$ lactose seemed to increase slightly while no clear trend could be observed for the other acidic oligosaccharides.

\section{Discussion}

Elephant milk and human milk are unique with regard to their content and variety of neutral and acidic lactosederived oligosaccharides compared with milk of any other

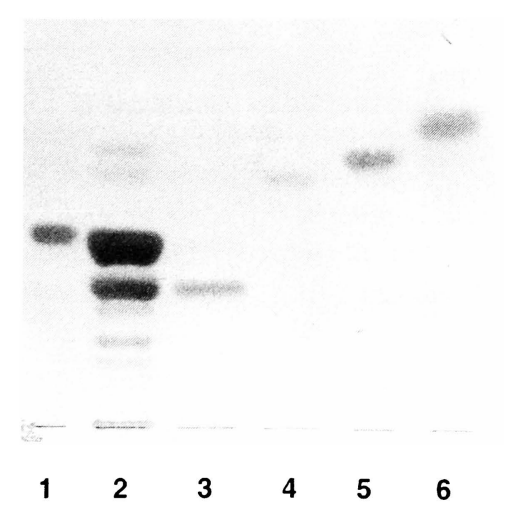

Fig. 5. Silica-high-performance TLC of fraction $E$ from elephant milk after Sephadex G25 gel filtration, containing a component which was later identified as $\alpha$-galactosyllactose. Lane designation: (1) lactose standard; (2) Sephadex G25 fraction E; (3) peak 2 in Fig. 4; (4) glucose standard; (5) galactose standard; (6) fucose standard. species. Considering that in human milk more than 130 components have been characterized so far, and many more can be expected, it is surprising that the oligosaccharide pattern in elephant milk is even more complex and the total concentration is even higher.

From HM it is known that there are marked differences in the total oligosaccharide content, especially between early and mature samples, as was shown a long time ago by Montreuil \& Mullet (1960). Quantitative data for individual components are difficult to obtain as more than 130 different oligosaccharides are present in human breast milk and are partly dependent on the Lewis blood group and secretor activity of the mother (Egge, 1993). In the present study, we found an increase in LNT in all milk samples analysed. However, in HM 1 and 2 the total concentration was about twice as much or even higher than in HM 3 and 4. Here, in the mature samples ( $>10 \mathrm{~d}$ post partum) the concentration of the Lewis a-active component LNFP II was about two to four times higher than that of LNT. This is an interesting observation as until now it has been believed that LNT is the major oligosaccharide in human milk. Although more investigations are needed we conclude from our findings that not only the pattern of some oligosaccharides but also their concentration depends on the blood group and secretor status of the donor.

There were also shifts in the sialylated oligosaccharides although no general trend was observed. The biological importance of the changing pattern of neutral and acidic oligosaccharides is unknown at present. Further interesting questions which cannot be answered at present are whether there are differences in the oligosaccharide pattern between fore, mid and hind milk and whether the type of the diet influences this pattern. Although there is no direct proof the general opinion is that the diet does not influence the milk carbohydrate content.

The question as to whether changes occur during lactation in elephant milk remains open as we have not been able to collect the appropriate samples. 


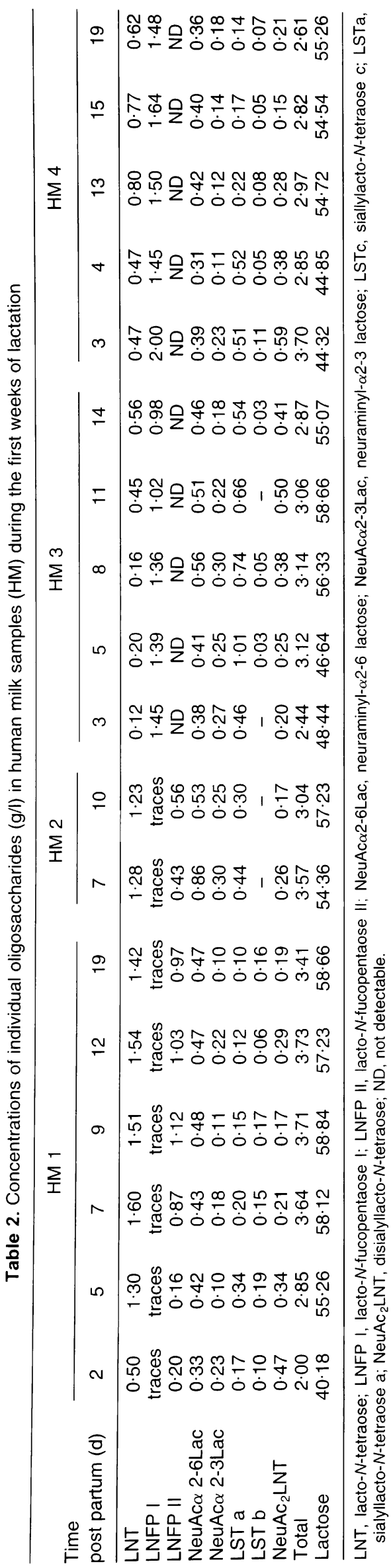

Milk samples from other species which we have investigated (e.g. cow, pig, horse, Barbary ape (Macaca sylvana) and rhesus monkey) showed considerably lower oligosaccharide concentrations compared with human milk (Kunz et al. 1996b; Braun, 1997; Rudloff et al. 1998) and elephant milk. In general, patterns were simple with only a few acidic components present. Monkey milk, however, is something of an exception, containing a variety of fucosylated oligosaccharides but in much lower concentrations $(0 \cdot 5-1 \mathrm{~g} / \mathrm{l})$ compared with human milk (5-8g/l; Kunz et al. $1996 b$ ). Milk carbohydrates of other eutherian species, e.g. Southern elephant seal (Mirounga leonina) (Carlini et al. 1994) and bears (Jenness et al. 1972), are reported to be mainly oligosacchrides. However, total concentrations are low, and simple small oligosaccharides dominate. In bear milk, lactose is only a minor carbohydrate (Jenness et al. 1972; Oftedal \& Iverson, 1995). However, when compared with human milk and elephant milk, bear milk has a rather simple carbohydrate pattern. The major component was found to be galactosyllactose (Oftedal \& Iverson, 1995) which is probably identical to that described in bear milk by Urashima et al. (1997). It has also been found in ovine, bovine and caprine colostrum (Urashima et al. $1989,1991)$ whereas in human milk it is only present in trace amounts.

In mammary secretions of marsupials (Messer \& Green, 1979), concentrations and pattern of oligosaccharides vary widely over the course of lactation with a maximum level and complexity in lactational stages where the young is permanently inside the pouch. Marsupial components show fundamentally different chemical structures compared with eutherian oligosaccharides (Messer et al. 1980; Urashima et al. 1994). In marsupials, chain elongation of lactose is achieved by the attachment of galactose units in $\beta 1-3$ linkage, catalysed by the enzyme lactose- $\beta 1$-3-galactosyltransferase (EC 2.4.1) which is not present in eutherian mammary tissue (Messer \& Nicholas, 1991). It is also worth noting, that in contrast to human milk and elephant milk oligosaccharides, none of the milks from marsupial types isolated so far contains any fucose residues.

Unexpectedly, most monotreme oligosaccharides do not follow metatherian, but eutherian design and also contain fucose (Messer \& Kerry, 1973). However. again, the pattern is simple with mainly fucosyl- and difucosyllactose present.

One of the core structures in elephant milk oligosaccharides seems to be lacto- $N$-neo-tetraose which can be consistently found in platypus milk whereas in human milk this LNT-type is only present in minor amounts. We found similar relations in milk from the rhesus monkey which also contains more lacto- $N$-neo-tetraose (Gal $\beta 1$-4GIcNAc $\beta 1-3 \mathrm{Gal} \beta 1-4 \mathrm{Glc})$ than LNT (Gal $\beta 1-3 \mathrm{GICNAc} \beta 1$ 3Gal $\beta 1-4 G 1 c$ ) (Kunz et al. 1996b).

The biological significance of the unique oligosaccharide composition of human milk and elephant milk remains to be elucidated. There is striking evidence that oligosaccharides in human milk are involved in neonatal host defence and similar components have been shown to affect endothelialleucocyte interactions (Springer, 1990; Zopf \& Roth, 1996; Kunz, 1999). In addition to this, milk oligosaccharides, along with lactose, may play a role in postnatal brain development (Sadhu, 1948; Carlson, 1985). Many newborn 
mammals undergo a period of rapid postnatal brain development which requires large amounts of glycolipids which are components in the cell membranes of neurones and myelin.

Galactocerebroside, with galactose as its polar head group. is the predominating glycolipid in myelin. The liver may not be capable of providing all the galactose needed by the young mammal during this period of myelination and brain development. Thus, a possible role of milk oligosaccharides in which galactose is a main component is that it may ensure that galactose levels in the infant do not become limiting during this time. A prerequisite for this mechanism is that oligosaccharides are not completely excreted via faeces but are to some extent absorbed in the digestive tract.

We recently addressed this question by applying stable isotopes in lactating women and demonstrated that orally applied $\left[{ }^{13} \mathrm{C}\right]$ galactose or $\left[{ }^{13} \mathrm{C}\right]$ glucose is directly incorporated into milk lactose and oligosaccharides (Kunz et al. 1999; Obermeier et al. 1999). Moreover, in subsequent studies we have shown that some of the in vivo labelled oligosaccharides were excreted in the infants " urine (C Kunz, S Obermeier, S Rudloff, G Pohlentz and MJ Lentze, unpublished results). These results confirm our previous studies comparing the urinary oligosaccharide pattern in breast-fed or formula-fed preterm infants (Rudloff et al. 1996). The conclusion is that some oligosaccharides must have been absorbed. Therefore, one cannot exclude the possibility that oligosaccharides, besides their local action. may also have systemic effects, e.g. on brain glycoconjugate composition. This suggestion is also confirmed by Carlson \& House (1986) who compared an intraperitoneal administration $v$. an intragastric application of neuraminic acid on rat brain composition. They found that both oral and intraperitoneal routes resulted in significantly more cerebral and cerebellar glycolipid and glycoprotein neuraminic acid than did glucose injection. Also, the advantage of orally given sialyllactose, the major acidic fraction in human milk, compared with free neuraminic acid on brain composition in rats has been shown by Witt et al. (1979). A further indication that dietary carbohydrates may be important for normal brain composition is the observation that in patients with classic galactosaemia exogenous galactose could be important to maintain a correct UDP-glucose : UDP-galactose ratio in some cells (Gibson et al. 1996). The impairment of UDP-galactose concentration in affected subjects could be responsible, in part, for the altered biosynthesis of brain glycolipids in these subjects (Petry et al. 1991).

It is well known that both species, man and elephant, show similar patterns of postnatal ontogeny; they both grow slowly, have relatively large and highly developed central nervous systems developing mainly after birth and show a high degree of intelligence and learned behaviour. Encephalization is considerably higher in man and elephant compared with non-human primates (e.g. rhesus monkey) reflecting the differences in milk oligosaccharide concentration (Braun, 1997). Thus, we speculate that lactosederived oligosaccharides, and in particular the galactose moeity, could play a role in the development of the infant brain.

\section{Acknowledgements}

We appreciate the FAB-MS and NMR analysis by Professor Dr H. Egge, Dr G. Pohlentz and Dr R. Hartmann at the Institute for Physiological Chemistry in Bonn (Germany).

\section{References}

Bergman HC \& Housley C (1968) Chemical analysis of American opossum (Didelphis virginiana) milk. Comparative Biochemistry and Phvisiology 25, 213-218.

Braun D (1997) Die Milchkompositionen der Säugetiere im Vergleich und die Milch des Menschen (Comparison of the milk composition of mammals and the milk of humans). $\mathrm{PhD}$ Thesis, University of Witten, Germany.

Carlini AR, Márquez MEI, Soave G, Vergani DF \& Ronayne de Ferrer PA (1994) Southern elephant seal, Mirounga leonina: composition of milk during lactation. Polar Biology 14, 37-42.

Carlson SE (1985) $\mathrm{N}$-acetylneuraminic acid concentrations in human milk oligosaccharides and glycoproteins during lactation. American Journal of Clinical Nutrition 41, 720-726.

Carlson SE \& House SG (1986) Oral and intraperitoneal administration of $N$-acetylneuraminic acid: effect on rat cerebral and cerebrellar $N$-acetylneuraminic acid. Journal of Nutrition 116, 881-886.

Dische Z (1962) General color reactions. In Methods in Carbohydrate Chemistry, vol. 1, pp. 488-494 [RL Whistler and ML Wolfrom, editors]. New York, NY: Academic Press.

Egge H (1993) The diversity of oligosaccharides in human milk. In New Perspectives in Infant Nutrition, pp. 12-26 [B Renner and G Sawatzki, editors]. Stuttgart: Thieme Verlag.

Egge H, Dell A \& von Nicolai H (1983) Fucose containing oligosaccharides from human milk. I. Separation and identification of new constituents. Archives of Biochemistry and Biophysics 224, 235-253.

Gibson JB, Berry GT, Palmieri MJ, Reynolds RA, Mazur AT \& Segal S (1996) Sugar nucleotide concentrations in red blood cells of patients on protein- and lactose-limited diets: effects of galactose supplementation. American Journal of Clinical Nutrition 63, 704-708.

Gross R \& Bolliger A (1958) The occurrence of carbohydrates other than lactose in milk of a marsupial. Trichosurus vulpecula. Australian Journal of Sciences 20, 184-185.

Hardy MR \& Townsend RR (1988) Monosaccharide analysis of glycoconjugates by anion exchange chromatography with pulsed amperometric detection. Analytical Biochemistry 170, 54-62.

Jenness R, Erickson AW \& Craighead JJ ( 1972) Some comparative aspects of milk from four species of bears. Journal of Mammalogy 53, 34-47.

Kobata A (1977) Milk glycoproteins and oligosaccharides. In The Glycoconjugates, vol. 1, pp. 423-440 [MI Horowitz and W Pigman, editors]. New York, NY: Academic Press.

Kunz C (1999) Microbial receptor analogs in human milk: structural and functional aspects. In Probiotics, Other Nutritional Factors, and Intestinal Microflora, Nestlé Nutrition Workshop Serie's, vol. 42, pp. 157-174 [LA Hanson and RH Yolken, editors]. Philadelphia, PA: Lippincott-Raven Publishers.

Kunz C, Obermeier S, Rudloff S, Hartmann R, Pohlentz G, Brösicke H \& Lentze MJ (1999) Stable isotope investigations of the biosynthesis of lactose and oligosaccharides in lactating women. FASEB Journal 13, A 254.

Kunz C \& Rudloff S (1993) Biological functions of oligosaccharides in human milk. Acta Paediatrica 82, 903-912.

Kunz C, Rudloff S, Hintelmann A, Pohlentz G \& Egge H (1996a) High-pH anion exchange chromatography with pulsed amperometric detection and molar response factors of human milk oligosaccharides. Journal of Chromatography. B 685, 211-221. 
Kunz C, Rudloff S, Pohlentz G \& Egge H (1996b) Oligosaccharides in milk of different species including man, rhesus monkey, cow and pig. FASEB Journal 10. A748.

Kunz C. Rudloff S, Pohlentz G, Lönnerdal B \& Egge H (1993) Sialylated and fucosylated oligosaccharides in rhesus monkey milk. FASEB Journal 7, A 823.

McCullagh KG, Lincoln HG \& Southgate DA (1969) Fatty acid com-position of milk fat of the Asian elephant. Nature 222, 493-494.

Messer M \& Green B (1979) Milk carbohydrates of marsupials. I. Quantitative and qualitative changes in milk carbohydrates during lactation in the tammar wallaby (Macropus eugenii). Australian Journal of Biological Sciences 32, 519-5.31.

Messer M \& Kerry KR (1973) Milk carbohydrates of the echidna and platypus. Science 180, 201-203

Messer M \& Mossop GS ( 1977 ) Milk carbohydrates of marsupials. 1. Partial separation and characterization of neutral milk oligosaccharides of the Eastern grey kangaroo. Australian Journal of Biological Sciences 30, 379-388.

Messer M \& Nicholas KR (1991) Biosynthesis of marsupial milk oligosaccharides: characterization and developmental changes of two galactosyltransferases in lactating mammary glands of the tammar wallaby, Macropus eugenii. Biochimica et Biophysica Acta 1077, 79-85.

Messer M. Trifonoff E. Stern W. Grant Collins J \& Howard Bradbury J (1980) Structure of a marsupial milk trisaccharide. Carbohydrate Research 83, 327-334.

Montreuil J (1993) The saga of human milk gynolactose. In New Perspectives in Infant Nutrition, pp. 3-11 [B Renner and G Sawatzki. editors]. Stuttgart: Thieme Verlag.

Montreuil J \& Mullet S (1960) Etude des variations des constituents glucidiques du lait de femme au cours de la lactation (Study of the variation in sugars of human milk during lactation). Bulletin de la Societe Chimique et Biologique 42, 365-377.

Obermeier S, Rudloff S. Brösicke H, Hartmann R \& Kunz C (1999) Analysis of ${ }^{13} \mathrm{C}$-enriched milk, urine and breath of a lactating woman after an oral $\left[1-{ }^{13} \mathrm{C}\right]$ galactose or $\left[1-{ }^{1.3} \mathrm{C}\right]$ glucose bolus. FASEB Journal 13, A254.

Oftedal OT \& Iverson SI (1995) Comparative analysis of nonhuman milks. A. Phylogenetic variation in the gross composition of milks. In Handbook of Milk Composition, pp. 749-789 [RG Jensen. editor]. San Diego, CA: Academic Press.

Petry K, Greinix HT, Nudelman E, Eisen H, Hakomori S-I, Levy HL \& Reichardt JKV (1991) Characterization of a novel biochemical abnormality in galactosemia: deficiency of glycolipids containing galactose or $N$-acetylgalactosamine and accumulation of precursors in brain lymphocytes. Biochemical Medicine and Metabolic Biology 46, 93-104.

Rudloff S, Braun D, Schad W \& Kunz C (1998) Oligosaccharides in milk - a relationship to brain development? FASEB Journal 12, A235.

Rudloff S, Pohlentz G, Diekmann L, Egge H \& Kunz C (1996) Urinary excretion of lactose and oligosaccharides in preterm infants fed human milk or infant formula. Acta Paediatrica $\mathbf{8 5}$, 598-603.

Sadhu DP (1948) Correlation between the lactose content of milk and the cerebroside and choline content of brain. Journal of Dairy Science 31, 347-351.

Springer TA (1990) Adhesion receptors of the immune system. Nature 346, 425-434.

Stahl B, Thurl S, Zeng J, Karas M, Hillenkamp F, Steup M \& Sawatzki G (1994) Oligosaccharides from human milk as revealed by matrix-assisted laser desorption/ionization mass spectrometry. Analytical Biochemistry 223, 218-226.

Svennerholm L (1957) Quantitative estimation of sialic acids. II. A colorimetric resorcin hydrochloric acid method. Biochimica et Biophisica Acta 24, 604-611.

Urashima T, Kusaka Y, Nakamura T, Saito T, Maeda N \& Messer M (1997) Chemical characterization of milk oligosaccharides of the brown bear. Ursus arctos yesoensis. Biochimica et Biophysica Acta 1334, 247-255.

Urashima T. Saito T, Nishimura J \& Ariga H (1989) New galactosyllacotse containing alpha-glycosidic linkage isolated from ovine (Booroola dorset) colostrum. Biochimica et Biophysica Acta 992. 375-378.

Urashima T, Saito T, Ohmisya K \& Shimazaki K (1991) Structural determination of three neutral oligosaccharides in bovine (Holstein-Friesian) colostrum, including the novel trisaccharide GaINAc $\alpha 1-3 \mathrm{Gal} \beta 1-4 \mathrm{G} 1 \mathrm{c}$. Biochimica et Biophysica Acta 1073, 225-229.

Urashima T, Saito T. Tsuji Y, Taneda Y, Takasawa T \& Messer M (1994) Chemical characterization of sialyl oligosaccharides isolated from tammar wallaby (Macropus eugenii) milk. Biochimica et Biophysica Acta 1200, 64-72.

Witt W, von Nicolai H \& Zilliken F (1979) Uptake and distribution of orally applied $\mathrm{N}$-acetyl- $\left({ }^{14} \mathrm{C}\right)$-neuraminyllactose and $\mathrm{N}$ acetyl- $\left({ }^{14} \mathrm{C}\right)$-neuraminic acid in the organs of newborn rats. Nutrition and Metabolism 23, 51-61.

Zopf D \& Roth S (1996) Oligosaccharide anti-infective agents. Lancet 347, 1017-1021. 